\title{
Pescadores Artesanais do Litoral de Alagoas: Socioeconomia e Acidentes de Trabalho Envolvendo Organismos Marinhos
}

Artisanal Fishermen of Alagoas Coast: Socioeconomics and Work Accidents Involving Marine Organism

Los Pescadores Artesanales, Playa, Alagoas: Socioeconomía y Accidentes de Trabajan con Organismos Marinos

Elíne Monteiro Calazans ${ }^{1}$

Elaine Martins Silva ${ }^{2}$

Josinete Silva de Liro ${ }^{3}$

Cláudio L. S. Sampaio ${ }^{4}$

\section{Resumo}

Objetivo: Descrever a socioeconomia dos pescadores artesanais do litoral alagoano e seus acidentes de trabalho envolvendo organismos marinhos. Método: A obtenção dos dados foi realizada seguindo os métodos qualiquantitativos:

(i) entrevistas

semiestruturadas, (ii) observações diretas, (iii) registros fotográficos. $\mathrm{O}$ estudo foi realizado entre 2013 e 2015 , a amostra foi 168 entrevistados, selecionados através da técnica bola de neve. Resultado: Dos pescadores, 97\% são homens, com baixo nível de instrução, experiência profissional média de 27,7 \pm 13 anos e jornada diária de trabalho média de $14 \pm 5$ horas. Registraram-se 1.242 acidentes: 752 por peixes, 345 cnidários e equinodermos e 145 por materiais de pesca. Os bagres (Ariidae) foram responsáveis por 54\% dos acidentes, ferroadas $(77,7 \%)$ foi o

${ }^{1}$ Engenheira de Pesca. Formada pela UFAL. Autora correspondente: Av. Beira Rio, s/n. Centro. 57200000, Penedo, AL, Brasil. E-mail: elinecalazans@gmail.com

${ }^{2}$ Graduanda em Engenharia de Pesca pela Universidade Federal de Alagoas (UFAL).

${ }^{3}$ Graduanda em Engenharia de Pesca pela Universidade Federal de Alagoas (UFAL)

${ }^{4}$ Doutor em Zoologia pela Universidade Federal da Paraíba. Docente do curso de Lic. Ciências Biológicas e Engenharia de Pesca da UFAL, Unidade Educacional Penedo. Laboratório de Ictiologia e Conservação.

Recebido: Abr./2018 - Aceito: Ago./2018. 
Calazans EM, Silva EM, Liro JS, Sampaio CLS Pescadores Artesanais do Litoral de Alagoas: Socioeconomia e Acidentes de Trabalho Envolvendo Organismos Marinhos

ferimento mais frequente, sobretudo nos membros superiores e inferiores (95,5\%). Dos entrevistados, 58\% afirmaram não utilizarem medida de proteção e apenas $39 \%$ procuraram tratamento médico, contudo registros sobre atendimentos às vítimas desses acidentes nas Unidades de Saúde visitadas são inexistentes. Dos acidentados, 62,4\%, afastaram-se do trabalho por período de um a quatro meses, destacando-se um caso de invalidez. Conclusão: Os resultados obtidos reforçam a necessidade de medidas que estimulem o uso de equipamentos de proteção e a qualificação dos profissionais de saúde, visando melhoria do atendimento e notificação destes acidentes.

\section{Descritores:Fatores Socioeconômicos;}

Riscos Ocupacionais; Saúde do Trabalhador.

\section{Abstract}

Objective: To describe the socioeconomic of artisanal fishermen the coast of Alagoas and know the work accidents involving marine fauna. Methods: Data collection was performed following the qualitativequantitative methods: (i) semi- structured interviews, (ii) direct observations, (iii) photographic records. The study was conducted between 2013 and 2015, the sample was 168 interviewed, selected through the snowball sampling technique. Results:

Of the fishermen, 97\% are men, adults, with low levels of education, fishing average experience mean of $27.7 \pm 13$ years, and working day of $14 \pm 5$ hours. There were 1,242 accidents recorded: 752 with fish, 345 cnidarians and echinoderms, and 145 by fishing materials. The catfish (Ariidae) were responsible for $54 \%$ of accidents, and stings $(77.7 \%)$ were more frequent, especially in the upper and lower limbs $(95.5 \%)$.

Of the interviewees, $58 \%$ said they did not use protection measure. Only 39\% sought medical treatment. Records of care for victims of diseases in the visited hospitals are non-existent. Of the victims, $62.4 \%$ retired from work for a period of one month. Highlighting a case of total disability. Conclusion: The results obtained reinforce the need for measures that stimulate the use of protective equipment and the search of health professionals, the direction of care and the recording of errors. 
Calazans EM, Silva EM, Liro JS, Sampaio CLS Pescadores Artesanais do Litoral de Alagoas: Socioeconomia e Acidentes de Trabalho Envolvendo Organismos Marinhos

\section{Descriptors: Nutritional Status; Anthropometry; Chronic Renal Insufficiency.}

\section{Resumen}

Objective: To describe the socioeconomic of artisanal fishermen the coast of Alagoas and know the work accidents involving marine fauna. Methods: Data collection was performed following the qualitativequantitative methods: (i) semistructured interviews, (ii) direct observations, (iii) photographic records. The study was conducted between 2013 and 2015, the sample was 168 interviewed, selected through the snowball sampling technique. Results:

Of the fishermen, 97\% are men, adults, with low levels of education, fishing average experience mean of $27.7 \pm 13$ years, and working day of $14 \pm 5$ hours. There were 1,242 accidents recorded: 752 with fish, 345 cnidarians and echinoderms, and 145 by fishing materials. The catfish (Ariidae) were responsible for $54 \%$ of accidents, and stings $(77.7 \%)$ were more frequent, especially in the upper and lower limbs (95.5\%).

Of the interviewees, $58 \%$ said they did not use protection measure. Only 39\% sought medical treatment. Records of care for victims of diseases in the visited hospitals are non-existent. Of the victims, $62.4 \%$ retired from work for a period of one month. Highlighting a case of total disability. Conclusion: The results obtained reinforce the need for measures that stimulate the use of protective equipment and the search of health professionals, the direction of care and the recording of errors.

Descriptores: Socioeconomic Factors; Occupational Risks; Occupational Health.

\section{Introdução}

A pesca artesanal é uma das atividades cujas condições de trabalho são bastante precárias, deixando os profissionais expostos a riscos de acidentes e doenças ${ }^{(1)}$.

As principais causas de acidentes de trabalho em pescadores são os ferimentos causados por animais aquáticos ${ }^{(2)}$. Outros agravos relatados por pescadores são ferimentos gerados por instrumentos de trabalho que podem ocasionar o afastamento do trabalhador de suas atividades.

Em Alagoas, a pesca artesanal exerce um importante papel 
Calazans EM, Silva EM, Liro JS, Sampaio CLS Pescadores Artesanais do Litoral de Alagoas: Socioeconomia e Acidentes de Trabalho

Envolvendo Organismos Marinhos

socioeconômico na geração de renda, sendo fundamental para a economia das comunidades litorâneas ${ }^{(3)}$. Todavia, apesar da importância, esse setor da economia regional recebe pouca atenção dos segmentos sociais e políticos incumbidos de executar o planejamento socioeconômico da região ${ }^{(4)}$.

Estudos recentemente da socioeconomia e saúde dos pescadores do sururu, Mytella falcata, em Maceió $(\mathrm{AL})^{(4)}$, registraram os acidentes de trabalho mais frequentes, sobressaindo, aqueles provocados pela fauna aquática. $\mathrm{O}$ estudo realizado no estado de Alagoas, por estes autores, revela o impacto negativo desses acidentes na vida econômica e social dos trabalhadores.

Como já amplamente relatado na literatura especializada ${ }^{(5)}$, não existem dados sobre a extensão dos problemas ambientais, econômicos e sociais causados por acidentes envolvendo organismos marinhos no Brasil. A falta de informações disponíveis e atualizadas dificulta uma análise dos acidentes, das espécies envolvidas e dos prejuízos socioeconômicos gerados, especialmente nas atividades artesanais, fatos que reforçam a importância do presente estudo. Neste contexto, este estudo teve como objetivo descrever a socioeconomia dos pescadores artesanais do litoral de Alagoas, bem como os acidentes de trabalho envolvendo organismos marinhos e materiais de pesca.

\section{Método}

O litoral do Estado de Alagoas possui aproximadamente $230 \mathrm{~km}$ de linha de costa, correspondendo a 2,9\% do litoral brasileiro, incluindo três regiões litorâneas (Norte, Central e Sul) (4). O presente estudo foi desenvolvido entre março de 2013 a dezembro de 2015, em 11 comunidades pesqueiras do Estado (Figura 1).

A obtenção dos dados foi realizada seguindo os métodos qualiquantitativos: (i) entrevistas semiestruturadas, (ii) observações diretas, (iii) registros fotográficos. Os trabalhos de campo nas comunidades eram constituídos de contatos com pescadores nos pontos de desembarque, nas colônias de pesca e com profissionais da saúde. Os pescadores foram selecionados a partir da técnica de amostragem snowball sampling (6), neste método um informante culturalmente competente recomenda outro de competência similar, repetindo o processo a partir dos novos incluídos. 
Os acidentes foram classificados em ferimentos puntiformes (ferroadas e/ou perfurações), caracterizado por dor de intensidade variável e necrose ocasional, em função das propriedades necróticas e neurotóxicas dos venenos; queimaduras (dependendo da ação dermonecrótica e neurotóxicas do veneno, podendo apresentar manifestação por placas lineares ou arredondadas eritematosas e dor intensa local instantânea) e ferimentos lacerados (dor de intensidade variável e lesões laceradas, podendo apresentar infecções secundárias) ${ }^{(7)}$. Sempre que possível, cicatrizes e ferimentos foram fotografados. As espécies envolvidas nos acidentes foram determinadas com auxílio de bibliografia especializada ${ }^{(8)}$.

Figura 1. Localização das Comunidades Estudadas no Litoral Alagoano

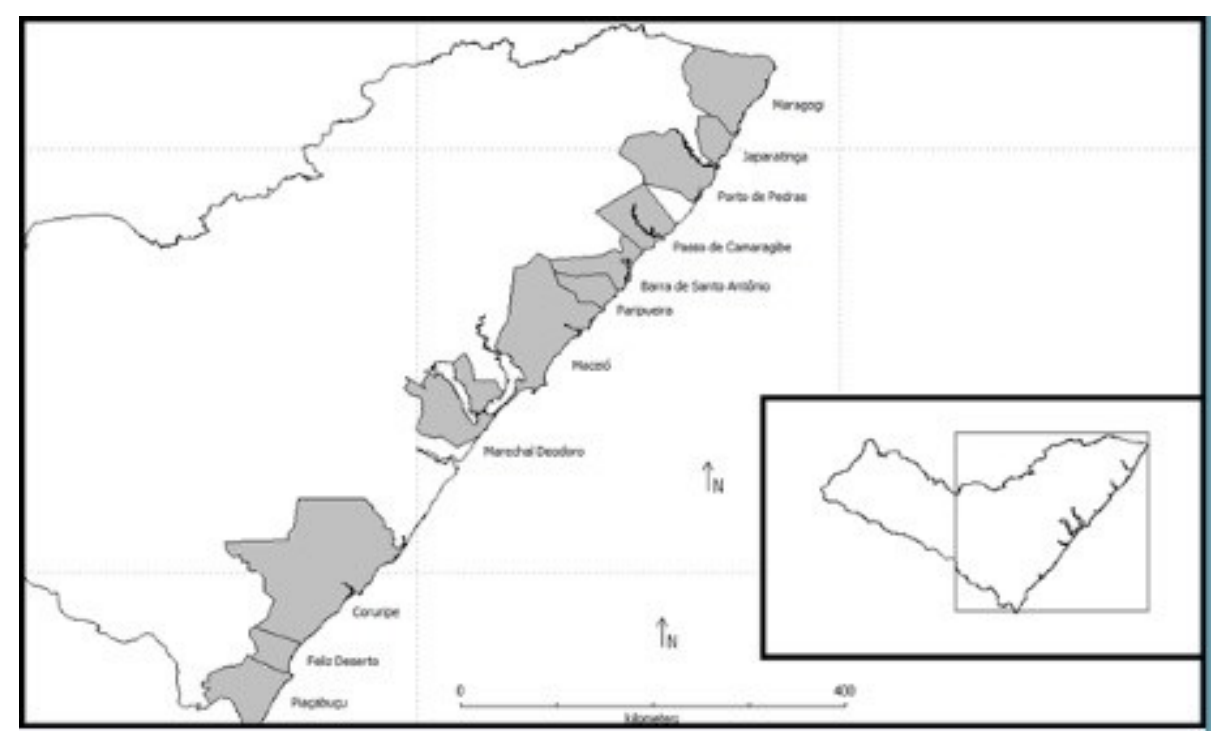

O banco de dados foi estruturado, com as fotografias armazenadas em arquivos digitais. Os resultados foram analisados por meio de estatísticas descritivas através de distribuições absolutas, relativas, percentuais e todas as médias estão seguidas do seu desvio-padrão. Realizou também o teste estatístico do Qui quadrado $\left(\chi^{2}\right)$, com $p=0,05$. O projeto que originou este artigo foi aprovado pelo Comitê de Ética em Pesquisa (parecer $\mathrm{n}^{\mathrm{o}}$ 2.508.811), seguindo a Resolução Ética 466/2012.

\section{Resultados}

Descrição Socioeconômica dos Pescadores 
Foram realizadas 168 entrevistas junto aos pescadores do litoral alagoano nos municípios (colônias de pesca) Maragogi (Z-15), Japaratinga (Z-28), Porto de Pedras (Z-25), Barra do Camaragibe (Z-22), Barra de Santo Antônio (Z-04), Paripueira (Z-21), Maceió (Z-01), Marechal Deodoro (Z06), Pontal do Coruripe (Z-10), Feliz Deserto (Z-37) e Pontal do Peba (Z-27).

$$
\text { Entre os pescadores }
$$
entrevistados, a maioria era do sexo masculino (97\%), com idades entre de 18 e 73 anos (média de 46,4 \pm 13 anos) e com escolaridade correspondendo a analfabeto $\quad(13,6 \%), \quad$ fundamental incompleto (62\%), fundamental completo $(9,5 \%)$, médio incompleto $(9,5)$, médio completo $(4,8 \%)$ e superior incompleto $(0,6 \%)$.

Com alusão à renda familiar obtida na pesca, em salários mínimos (SMs), considerando aquele referente ao ano de 2015 (R\$ 788,00 reais), observou-se que $49,4 \%$ dos pescadores afirmaram receber valores inferiores a um SM e 44\% entre um e dois SM. Em relação a fonte de renda dos entrevistados, 83,3\% afirmaram que a pesca artesanal é sua única atividade, e que desta advém o sustento de suas famílias. Todavia, 16,7\%, revelam que praticam, em paralelo com a pescaria, outras atividades remuneradas, com o intuito de incrementar o rendimento familiar. Ao serem questionados sobre o tipo de residência, $71,4 \%$ declararam que residem em imóvel próprio, 20,2\% alugado e $8,3 \%$ em imóvel dos pais ou outros familiares.

Quanto à composição familiar, $56 \%$ são compostas por até quatro membros e $44 \%$ por até nove componentes. Ressalta-se que durante as entrevistas a maioria dos pescadores relatou não desejar que seus filhos adentrem no setor pesqueiro.

\section{Fatores Relacionados à Vida Profissional}

O tempo de exercício na atividade pesqueira foi bastante amplo, variando de dois a 67 anos, com média ( \pm desvio padrão) de $27,7 \pm 13$ anos e $45,8 \%$ com menos de 30 anos na atividade pesqueira (Figura 2).

Quando questionados sobre o porquê da escolha da profissão, os resultados obtidos apontaram que $61,3 \%$ por falta de opção, enquanto $24,4 \%$ relataram que quiseram seguir a carreira do pai. Já 14,3\% afirmaram gostar da atividade e por isso resolveram ingressar. Ressalta-se que cada entrevistado apresentou mais de 
uma razão para ter entrado no setor

pesqueiro.

Figura 2. Frequência Relativa da Classe de Tempo de Atuação na

Atividade Pesqueira

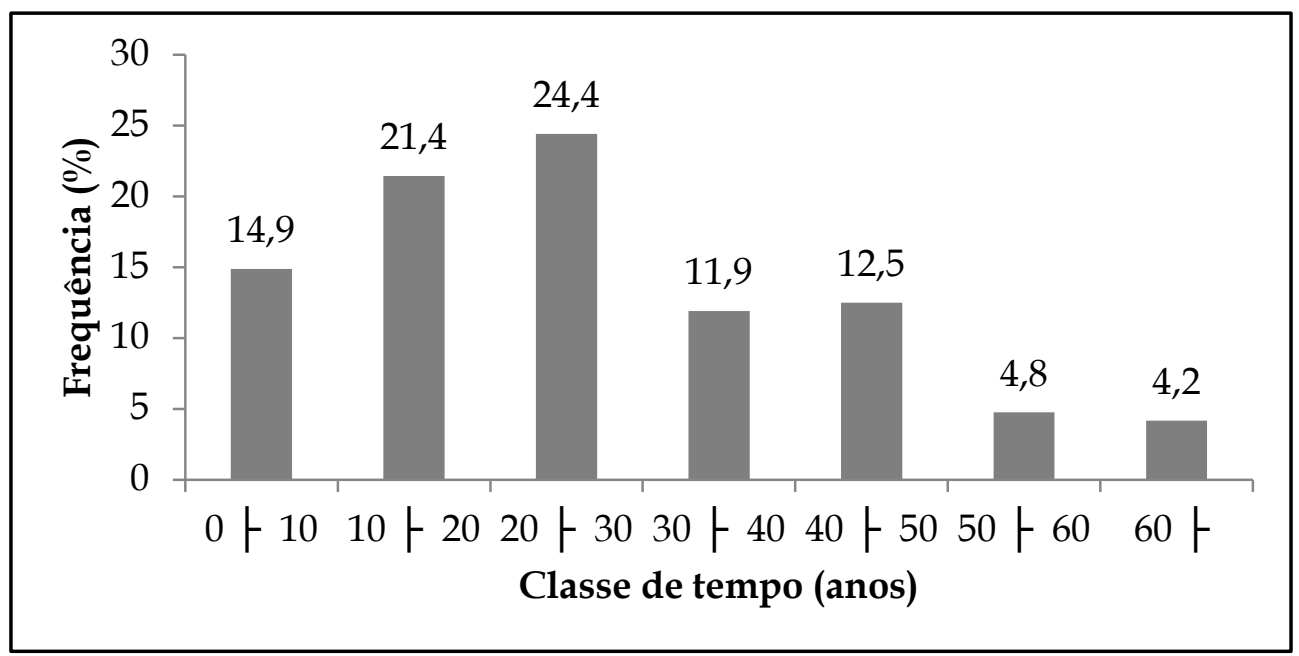

Em relação à jornada de trabalho, a maioria deles informou trabalhar de 3 a 21 horas por dia da profissão e dos riscos aos quais estão suscetíveis.

(79,2\%). Em algumas localidades (Paripueira, Barra de Santo Antônio, Japaratinga, Pontal do Coruripe e Pontal do Peba), os pescadores afirmaram que permanecem no mar durante até 15 dias $(20,8 \%)$. No que diz respeito às dificuldades enfrentadas no setor pesqueiro local, os pescadores relataram basicamente problemas como más condições de trabalho e ausência de políticas públicas voltadas à melhoria da infraestrutura e segurança. Dos entrevistados, $64,3 \%$ afirmaram que estão descontentes com a atividade pesqueira, em razão da desvalorização

\section{Sobre os Aspectos Relacionados aos Acidentes de Trabalho Envolvendo Organismos Marinhos e a Segurança dos Pescadores}

Todos os entrevistados relataram ter algum tipo de ferimento envolvendo animais marinhos, sendo geralmente ocasionado pelo contato com estruturas que estão presentes no corpo do animal e que são capazes de provocar lesões.

A maioria dos pescadores entrevistados (78\%) foi acometida por dois ou mais acidentes, registrando-se um total de 1.242 casos, sendo 752 envolvendo peixes, 345 organismos 
marinhos (cnidários e equinodermos) e os demais (145), causados por petrechos e materiais utilizados na pesca. O maior número de acidentes foi registrado na região litorânea Norte $(63,1 \%)$, seguida pelo litoral Sul $(25,6 \%)$ e Central $(11,3 \%)$.

O maior número de acidentes envolvendo peixes foi registrado na comunidade pesqueira Pontal do Coruripe (22\%), seguido por Paripueira (19\%), Barra de Santo Antônio (12,1\%), Barra do Camaragibe (11,6\%), Japaratinga (7,6\%), Maragogi (6,4\%), Porto de Pedras (6,1\%), Pontal do Peba (5,9\%), Feliz Deserto (3,7\%), Maceió (2,9\%) e Marechal Deodoro $(2,7 \%)$ (Tabela 1$)$.

Tabela 1. Distribuição dos Acidentes Envolvendo as Famílias dos Peixes e as Comunidades Pesqueiras no Litoral Alagoano

\begin{tabular}{|c|c|c|c|}
\hline & Bagre & Niquim & Arraia \\
\hline Tabagismo & Ariidae & Batrachoididae & Dasyatidae \\
\hline LOCALIDADES & $\mathrm{N}$ & $\%$ & $\mathrm{~N}$ \\
\hline Maragogi & 18 & 4,4 & 4 \\
\hline Japaratinga & 37 & 9,1 & 6 \\
\hline Porto de Pedras & 33 & 8,1 & 1 \\
\hline Barra do Camaragibe & 35 & 8,6 & 27 \\
\hline Barra de Sto Antônio & 76 & 18,7 & 2 \\
\hline Paripueira & 74 & 18,2 & 6 \\
\hline Maceió & 12 & 2,9 & 0 \\
\hline Marechal Deodoro & 5 & 1,2 & 2 \\
\hline Pontal do Coruripe & 88 & 21,6 & 6 \\
\hline Pontal do Peba & 13 & 3,2 & 10 \\
\hline \multirow[t]{3}{*}{ Feliz Deserto } & 19 & 4,7 & 0 \\
\hline & Bagre & Niquim & Arraia \\
\hline & Ariidae & Batrachoididae & Dasyatidae \\
\hline LOCALIDADES & $\mathrm{N}$ & $\%$ & $\mathrm{~N}$ \\
\hline Maragogi & 18 & 4,4 & 4 \\
\hline Japaratinga & 37 & 9,1 & 6 \\
\hline Porto de Pedras & 33 & 8,1 & 1 \\
\hline Barra do Camaragibe & 35 & 8,6 & 27 \\
\hline Barra de Sto Antônio & 76 & 18,7 & 2 \\
\hline Paripueira & 74 & 18,2 & 6 \\
\hline Maceió & 12 & 2,9 & 0 \\
\hline Marechal Deodoro & & & \\
\hline
\end{tabular}

As espécies mais citadas pelos pescadores foram: Bagre (Ariidae, 54\%), Arraia (Dasyatidae, 9\%) Niquim
(Batrachoididae, 8,5\%) e Beatriz (Scorpaenidae, 6\%). 
Calazans EM, Silva EM, Liro JS, Sampaio CLS Pescadores Artesanais do Litoral de Alagoas: Socioeconomia e Acidentes de Trabalho Envolvendo Organismos Marinhos

Os bagres pertencentes à família Ariidae foram os maiores responsáveis pelos acidentes em todas as localidades do litoral alagoano. Os acidentes ocorreram principalmente quando os pescadores estão manuseando e/ou quando pisam nos exemplares durante a atividade pesqueira.

Quanto aos acidentes por cnidários (caravela - Physaliidae), a maior incidência foi registrada na comunidade pesqueira de Paripueira $(49,1 \%)$, seguido por Pontal do Peba (20,2\%) e Marechal Deodoro (13,3\%). Para os ouriços do mar, os maiores números foram registrados em Paripueira (51,2\%), Marechal Deodoro
$(35,5 \%)$ e Barra de Santo Antônio (7,0\%). Estes acidentes ocorrem predominantemente durante o trajeto realizado pelos pescadores até as embarcações e/ou durante a atividade pesqueira nos recifes costeiros.

Em relação aos tipos de lesões, os ferimentos puntiformes (ferroadas) foram os mais frequentes entre os acidentes registrados $(77,7 \%)$, e foram causados por animais que apresentam ferrão, como arraia, bagre, niquim e beatriz (Figura 3. A e B), que ao penetrarem nos tecidos da vítima, liberam a peçonha do tecido glandular que os recobrem, causando dor intensa.

Figura 3. A - Ferroada Causada por Arraia (Dasyatidae); B - Ferroada por Peixe Beatriz (Scorpaenidae) na Perna do Pescador

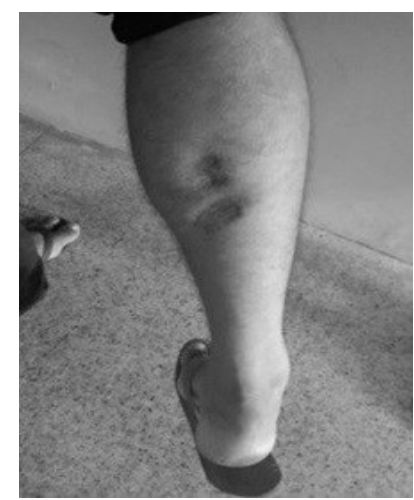

Quanto aos

ferimentos puntiformes (perfurações) causados por raios presentes nas nadadeiras dos peixes, estes corresponderam a $20,8 \%$ dos casos de acidentes, ocorridos quanto

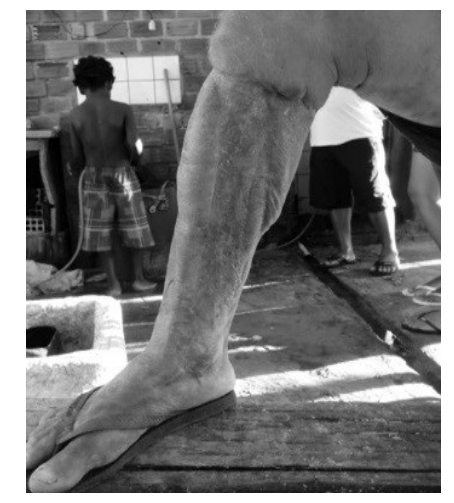

aos ferimentos puntiformes (perfurações) causados por espinhos presentes nas nadadeiras dos peixes, estes corresponderam a $20,8 \%$ dos casos de acidentes, ocorridos geralmente 
Calazans EM, Silva EM, Liro JS, Sampaio CLS

durante o manuseio do pescado depois da atividade de pesca. Os ferimentos lacerados (mordidas) representaram $1,6 \%$ e foram causados por animais que mordem, como a moreia (Muraenidae). Acidentes por materiais e apetrechos de pesca como anzóis, facas e nylon, bem como por equipamentos da embarcação também foram registrados (guincho e escape).

Comparando a faixa etária de idade com a porcentagem dos acidentes sofridos pelos pescadores e tempo de atuação relacionado ao número de acidentes observou-se que não foram significativas. $\left(\chi^{2}=0,99 ; p>0,05\right),\left(\chi^{2}=\right.$ $1,00 ; p>0,05)$, respectivamente.

Em relação à ocasião dos acidentes, estes ocorreram predominantemente durante as atividades de pesca, sobretudo quando estavam despescando e/ou manipulando o pescado $(67,8 \%)$. O relato de acidentes por pescadores que estavam manipulando equipamentos de pesca $\begin{array}{llll}\text { aconteceu } & \text { em } & 19,2 \% & \text { dos }\end{array}$ acontecimentos. Acidentes em pescadores que operavam ou conduziam embarcações e preparavam iscas foram relatados em $13 \%$ dos casos.

As regiões anatômicas mais atingidas pelas lesões, segundo os entrevistados, foram os membros superiores $(48,7 \%)$, os membros inferiores $(46,8 \%)$ e outras partes do corpo $(12,5 \%)$.

Todos os entrevistados alegaram sentir dor ao sofrer qualquer tipo de lesão, dos quais $71,4 \%$ afirmaram sentir moderada a muito forte nos diferentes tipos de lesão, sejam laceradas ou puntiformes. Todas as vítimas também sofreram os principais sintomas pertinentes a acidentes com animais marinhos (dor intensa, febre, dormência, edema e necrose).

Quanto à procura por atendimento médico, apenas 39\% procuraram alguma Unidade de Saúde, geralmente ao terem complicações ou sofrerem acidentes por arraia e niquim, que ao ferroar libera toxinas que podem provocar sintomas mais graves. Dentre os $61 \%$ dos profissionais que não procuraram tratamento médico, 55,9\%, realizaram algum tipo de tratamento popular na tentativa de curar a lesão ou ao menos amenizar a dor e 5,1\% automedicaram-se.

Apesar do grande número de acidentes, os profissionais da saúde que foram entrevistados $(n=6)$ encontram-se desinformados quanto aos registros de acidentes envolvendo organismos marinhos nas unidades visitadas, sugerindo a subnotificação dos 
Calazans EM, Silva EM, Liro JS, Sampaio CLS Pescadores Artesanais do Litoral de Alagoas: Socioeconomia e Acidentes de Trabalho Envolvendo Organismos Marinhos

acidentes e aos procedimentos adequados, informando que o tratamento empregado nesses casos é semelhante a outros tipos de acidentes, sendo tratados apenas de forma sintomática.

Com relação ao tempo de incapacidade do pescador para o trabalho, em decorrência dos acidentes, $37,6 \%$ dos entrevistados afirmaram não ter tido problemas para desenvolver suas atividades laborais. No entanto, $24,8 \%$ relataram ter ficado impossibilitado de trabalhar por até sete dias, por terem sofrido lesões de menor gravidade e pelo fato de necessitarem trabalhar para manter suas famílias. Os que tiveram necessidade de ficar até 16 meses afastados do trabalho (37\%), alegaram ter causado sofrimento a seus familiares e prejuízos econômicos. Destaca-se um caso de incapacidade permanente de um pescador acidentado por arraia (Dasyatidae) (0,6\%) (Figura 5. A e B).

Figura 4. A - Cicatriz de Ferroada por Arraia (Dasyatidae); B - Pescador Incapacitado a Voltar as Atividades Laborais por Consequência do Acidente

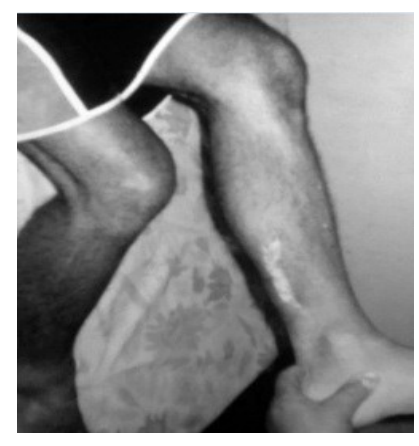

Os Equipamentos de Proteção Individual (EPI) estão descritos no item 6.1 da Norma Regulamentadora (NR) $n^{\circ}$ 6 como "todo dispositivo ou produto, de uso individual utilizado pelo trabalhador, destinado à proteção de riscos suscetíveis de ameaçar a segurança e a saúde no trabalho". Quanto à utilização das medidas de proteção, no presente estudo, todos os

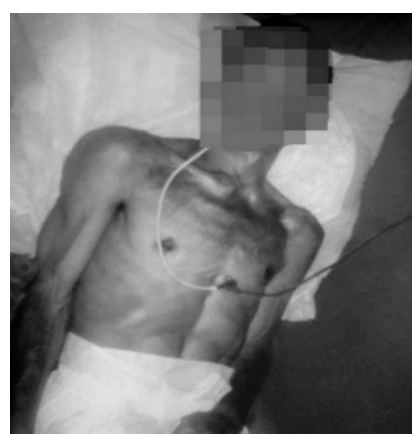

entrevistados afirmam que a atividade de pesca oferece riscos, contudo, 57,7\% (Tabela 7) destes ignoram a utilização EPI's.

Conquanto a maioria dos entrevistados $(54,8 \%)$ tenha afirmado ter recebido algum treinamento sobre primeiros socorros, 50,6\% admitiram não saber realizar qualquer tipo de procedimento e somente $45,2 \%$ 
responderam que sabem realizar algum procedimento de primeiros socorros.

\section{Discussão}

No litoral alagoano, os pescadores artesanais estão representados, em sua maioria, por homens. Esta realidade é comum aos trabalhadores vinculados à prática pesqueira artesanal (3). Este predomínio ocorre em virtude do esforço físico que a atividade exige, dos riscos oferecidos, e, sobretudo, pela forte discriminação cultural ainda existente. Quanto à idade o grupo etário predominante foi entre 30 e 49 anos, indicando um processo de senilização das comunidades pesqueiras, reflexo da falta de interesse dos jovens pela profisssão, atrelado ao desencorajamento por parte de seus pais e familiares ${ }^{(9)}$.

A baixa escolaridade é típica dos praticantes da atividade da pesca artesanal, uma vez que, o baixo grau de instrução, pode estar atrelado à falta de tempo e à necessidade de trabalhar longos períodos para garantir o sustento da família ${ }^{(3,4)}$.

A renda mensal declarada pelos entrevistados é comparável à de pescadores que atuam em outras regiões da costa brasileira, apontando que a remuneração obtida nesta atividade é geralmente baixa ${ }^{(10)}$. No que se refere ao tipo de moradia, os resultados encontrados podem estar relacionados ao tempo em que os pescadores residem nas comunidades estudadas ${ }^{(4,3)}$.

Quanto ao tempo de atuação na pesca, aproxima-se do encontrado na capital alagoana ${ }^{(20)}$, onde $48 \%$ dos entrevistados afirmaram trabalhar na pesca a menos de 30 anos. Já aos motivos que os levaram a escolha da profissão, grande parte afirmou ter adentrado na atividade por não vislumbrar uma melhor perspectiva de vida $^{(2)}$.

Em relação a jornada de trabalho, foram registrados ritmos extenuantes, $\mathrm{o}$ que parece ser um padrão ao longo do litoral brasileiro ${ }^{(11)}$. A produção reduzida, acarreta um maior esforço de pesca e, consequentemente, em mais horas de exposição à atividade laboral (4).

Análises recentes dos dados do Sistema de Informação de Agravos de Notificação - SINAN ${ }^{(12)}$, no estado de Alagoas, indicaram que os acidentes mais registrados foram os causados por peixe sapo (Batrachoididae, 62,4\%), diferindo dos nossos resultados, onde a maioria dos acidentes foi ocasionada por bagres (Ariidae, 54\%). Essa diferença pode estar relacionada a maior 
amplitude do presente estudo, bem como do hábito dos pescadores em tratar esses ferimentos, evitando o atendimento médico.

Os acidentes envolvendo os bagres foram mais frequentes, sendo a potência do veneno variando com a espécie e o gênero, mas todos apresentam proteínas que provocam intensa dor local ${ }^{(13)}$, impossibilitando os pescadores de desenvolver suas atividades e gerando dificuldades econômicas.

As queimaduras por cnidários são comuns em todo mundo, inclusive ao longo da costa brasileira (14). Esses acidentes podem estar relacionados aos hábitos desses animais, que são discretos e podem passar despercebidos dos pescadores. A ação do veneno pode causar efeitos tóxicos e alérgicos e até mesmo a morte ${ }^{(15)}$.

Os ouriços existem ao longo de toda a costa e provocam acidentes traumáticos em grande número, sendo responsáveis por cerca de $50 \%$ dos acidentes nas cidades litorâneas ${ }^{(16)}$. O ambiente recifal raso e adjacente aos pontos de desembarque da produção pesqueira pode ser um dos motivos do elevado número de acidentes. Os sintomas decorrentes desses acidentes incluem parestesia, dor irradiada, hipotensão, fraqueza muscular, dentre outros ${ }^{(17)}$.

$\mathrm{Na}$ região Norte alagoana as principais artes de pesca utilizadas são: rede de emalhar, caceia e linha de mão, expondo os profissionais a um maior contato com esses organismos, tornando-os mais vulneráveis aos acidentes ${ }^{(18)}$. Na região Sul, onde a atividade pesqueira ser semi-industrial, tendo o camarão como principal alvo da pesca ${ }^{(19)}$. Já a região Central, apresenta uma maior atividade turística e menor pesqueira, devido as atividades paralelas que são desenvolvidas ${ }^{(3)}$.

Ferimentos provocados por animais ou causados pelos instrumentos de trabalho são comuns ${ }^{(2)}$, levando ao afastamento do trabalhador de sua atividade por dias ou meses.

$\mathrm{Na}$ Bacia do Alto Paraguai, Mato Grosso do Sul ${ }^{(20)}$, observou-se que não houve significância entre a faixa etária e a porcentagem de acidentes sofridos pelos pescadores corroborando com o resultado encontrado no presente estudo. Contudo, quanto ao tempo de atuação relacionada a porcentagem de acidentes, difere do resultado deste trabalho. Levando-se em consideração que no ambiente marinho há uma maior diversidade de organismos peçonhentos 
que no rio, as ocorrências de acidentes tornam-se mais frequentes.

Os membros superiores e inferiores são mais atingidos, devido o contato com os organismos marinhos através dos métodos de pesca, ficando sujeitos a riscos se não forem protegidos (21).

A maioria dos acidentados não procurou tratamento médico, contribuindo para a subestimação do número de acidentes. Esse fato pode estar relacionado a falta ou demora no atendimento, que para os pescadores, implica em perda de tempo, representando uma significativa perda econômica (4). Em muitos casos, realizaram algum tipo de tratamento popular ou automedicaram-se. A utilização desses tratamentos pode agravar ainda mais as lesões (21) e contribuir para o aumento da intensidade da dor e necroses, prejudicando, ainda mais, o quadro clínico.

Apesar do grande número de acidentes, os profissionais da saúde que foram entrevistados encontram-se desinformados quanto aos registros de acidentes envolvendo organismos marinhos nas unidades visitadas. Contudo, tais registros são obrigatórios no Sistema Nacional de Agravos de
Notificação (SINAN), uma vez que a análise dessas informações contribui para as ações de vigilância em saúde e na elaboração de políticas públicas.

Embora o tema acidentes venha sendo discutido, recentemente no Brasil, por vários autores $(12,22)$, à falta de informações e treinamento específico, para tratar esses acidentes, resultam na inaptidão dos profissionais de saúde ${ }^{(23)}$.

Cerca de $46 \%$ dos pescadores e $20 \%$ dos catadores de caranguejos da Baía de Guanabara (RJ) faziam uso de EPI's ${ }^{(24)}$, diferente das comunidades do litoral de Alagoas, onde a maioria $(57,7 \%)$ ignora seu uso. O uso adequado dos EPI's minimiza os riscos de acidente no trabalho, sobretudo quando envolve manipulação de materiais perfurocortantes ${ }^{(25)}$.

\section{Conclusão}

Os resultados obtidos indicam a precariedade que os pescadores artesanais sofrem na sua labuta diária e revelam altos índices de acidentes de trabalho envolvendo organismos marinhos.

Confirmamos as dificuldades enfrentadas pelos pescadores artesanais do litoral de Alagoas, sendo possível afirmar que os riscos aos quais se 
submetem são potencializados por suas condições de trabalho e vida. O pouco conhecimento por parte dos pescadores em relação aos primeiros socorros, prevenção dos acidentes, baixo grau de instrução, pouco acesso à saúde e baixa qualidade de vida são agravados pela excessiva jornada de trabalho.

Neste contexto, reforça-se a necessidade de maior atenção para a subnotificação dos acidentes com organismos marinhos. Do mesmo modo, medidas que estimulem o uso de EPI pelos pescadores e a qualificação dos profissionais de saúde deve ser encorajada, com vistas à redução do absenteísmo e à melhoria do atendimento e da notificação destes acidentes.

\section{Referências}

1. Silva GC, Sabino J, Alho CJR, Nunes LB, Haddad JV. 2010 Injuries and envenoming by aquatic animals in fishermen of Coxim and Corumbá municipalities, State of Mato Grosso do Sul, Brazil: identification of the causative agents, clinical aspects and first aid measures. Medicina Tropical (Minas Gerais) [periódico na Internet]. 2010 [citado em 02 de jan. 2018]; 43(5): 486-90. Disponível em: http://www.repositorio.

unb.br/bitstream/10482/10603/1/ARTI GO_InjuriesEnvenomingAquatic.pdf.
2. Garrone Neto D, Cordeiro CR, Haddad JV. Acidentes do trabalho em pescadores artesanais da região do Médio Rio Araguaia, Tocantins, Brasil.Saúde Pública (Rio de Janeiro) [periódico na Internet]. 2005 [citado 2018 jan. 12]; 21(3): 795-03. Disponível em: http://www.scielo. br/pdf/\%0D/csp/v21n3/13.pdf.

3. Santos EC, Sampaio CLS. A pesca artesanal na comunidade de Fernão Velho, Maceió (Alagoas, Brasil): de tradicional a marginal. Gestão Costeira Integrada (Santa Catarina) [periódico na Internet]. 2013 [citado em 03 jan. 2018]; 13(4): 513-24. Disponível em: http://www.scielo.mec.pt/pdf/rgci/v13n 4/v13n4a09.pdf

4. Tamano LTO, Araujo DM, Lima BBC, Silva FNF, Silva J. Socioeconomia e saúde dos pescadores de Mytella falcata da Lagoa Mundaú, Maceió-AL. Ciências Humanas (Belém) [periódico na Internet]. 2015 [citado em 20 de jan. de 2018]; 10(3): 699-10. Disponível em: http://www. scielo.br/pdf/bgoeldi/v10n3/1981-812 2-bgoeldi-10-3-0699.pdf

5. Haddad JV. Medical Emergencies Caused by Aquatic Animals: a zoological and clinical guide. New York: Springer; 2016

6. Biernacki P, Waldorf D. 1981 Snowball sampling-problems and techniques of chain referral sampling. Pesquisa (United States of America) [periódico na Internet]. 1981 [citado 2018 jan. 10]; 10(2):141-63. Disponível em: https://www.ncjrs. gov/App/Publications/abstract.aspx?ID $=146745$. 
7. Haddad JV. Animais aquáticos de importância médica no Brasil. Medicina Tropical (Minas Gerais) [periódico na Internet]. 2003 [citado em jan. 11]; 36(5): 591-97. Disponível em: http://www.scielo.br/pdf/rsbmt /v36n5/a09v36n5.pdf.

8. Haddad JV. Animais aquáticos potencialmente perigosos do Brasil. São Paulo: Roca; 2008.

9. D'incao F, Silva-Gonçalves R. Perfil socioeconômico e laboral dos pescadores artesanais de camarão-rosa no complexo estuarino de Tramandaí (RS), Brasil. Instituto de Pesca (São Paulo) [periódico na Internet]. 2016 [citado 2018 mar 20]; 42(2): 387-401. Disponível em: https://www.pesca.sp. gov.br/42_2_9BIP-1147p387-401.pdf.

10. Zacardi DM, Silva GS, Vaz EM, Silva LMA. Estudos dos aspectos sociais e técnicos da atividade pesqueira no município de Calçoene, Amapá, Extremo Norte do Brasil. Engenharia de Pesca (Maranhão) [periódico na Internet]. 2017 [citado em abr. 14]; 9(2):52-68. Disponível em: $\quad$ ppg.revistas.uema.br/index.php /REPESCA/article/download/1044/945

11. Pena GLP, Gomez CM. Saúde dos pescadores artesanais e desafios para a vigilância em saúde do trabalhador. Ciência e Saúde Coletiva (Rio de Janeiro) [periódico na Internet]. 2014 [citado em mar. 06]; 19(12): 46894698. Disponível em: http://www. scielo.br/pdf/csc/v19n12/pt_1413-8123 -csc-19-12-04689.pdf.
12. Reckziegel GC, Dourado FS, Garrone Neto D, Haddad JV. Injuries caused by aquatic animals in Brazil: an analysis of the data present in the information system for notifiable diseases. Medicina Tropical (Minas Gerais) [periódico na Internet]. 2015 [citado em jan. 04]; 48(4): 460-467. Disponível em: http:/www.scielo.br/ pdf/rsbmt/v48n4/1678-9849-rsbmt-4804-00460.pdf.

13. Cardoso JLC, França FOS, Wen FH, Malaque CMS, Haddad JV. Animais peçonhentos no Brasil: biologia, clínica e terapêutica dos acidentes, São Paulo: Sarvier; 2004.

14. Bastos Dmrf, Haddad JV, Nunes J. 2017 Human envenomations caused by Portuguese man-of-war (Physalia physalis) in urban beaches of São Luis City, Maranhão state, northeast coast of Brazil. Medicina Tropical (Minas Gerais) [periódico na Internet]. 2017 [citado 2018 abr. 15]; 50(1):130-134. Disponível em: http://www.scielo.br/ scielo.php?script $=$ sci_arttext\&pid $=\mathrm{S} 00$ 37-86822017000100130.

15. Neves RF, Amaral FD, Steiner AQ. Levantamento de registros dos acidentes com cnidários em algumas praias do litoral de Pernambuco (Brasil). Ciência e Saúde Coletiva (Rio de Janeiro) [periódico na Internet]. 2007 [citado em jan. 24]; 12(1): 231$237 . \quad$ Disponível em: http://www.scielo.br/pdf/csc/v12n1/22. pdf. 
16. Haddad JV. 2013 Dermatologia ambiental: Manifestações dermatológicas de acidentes por animais aquáticos (invertebrados). Dermatologia (Rio de Janeiro) [periódico na Internet). 2013 [citado em fev. 2018]; 88(4): 496-506. Disponível em: http://200.145.6.238/ bitstream/handle/11449/75830/2-s2.084884800673.pdf? sequence $=1 \&$ isAllo wed $=$ y.

\section{Haddad JV. Observation of initial} clinical manifestations and repercussions from the treatment of 314 human injuries caused by black sea urchins (Echinometra lucunter) on the Southeastern Brazilian coast. Medicina Tropical (Minas Gerais) [periódico na Internet]. 2012 [citado em jan. 14]; 45(3): 390-392. Disponível em: http://www.scielo.br/ pdf/rsbmt/v45n3/a21v45n3.pdf.

18. Souza CD, Batista VS, Fabré NN. Caracterização da pesca no extremo sul da Área de Proteção Ambiental Costa dos Corais, Alagoas, Brasil. Instituto de Pesca (São Paulo) [periódico na Internet]. 2012 [citado em 03 de jan. 2018]; 38(2): 155 - 169. Disponível em: https://www.pesca.sp.gov.br/38_ 2_155-169.pdf.

19. Santos MCF, Silva KCA, Cintra IHA. Carcinofauna acompanhante da pesca artesanal do camarão-sete-barbas ao largo da foz do rio São Francisco (Alagoas e Sergipe, Brasil). Fisheries and Aquatic Resources (Sergipe) [periódico na Internet]. 2016 [citado em 05 de jan. 2018]; 4(1)1-10. Disponível em: https://seer.ufs.br/ index.php/ActaFish/article/view/4853/ 4136.
20. Edilson AD. Análise do perfil socioeconômico, caracterização de acidentes e avaliação do acesso a informações sobre medidas preventivas de acidentes ocasionados por animais aquáticos a pescadores da Bacia do Alto Paraguai, Mato Grosso do Sul. Dissertação [Mestrado em Meio Ambiente e Desenvolvimento Regional] Universidade Anhanguera Uniderp; 2014 [citado em 15 de jan. 2018]; 1-72. Disponível em: https://s3.amazonaws.com/pgsskroton dissertacoes/1d1846f3de6ca7febd3d0c 607a9fa28f.pdf.

21. Silva GC, Sabino J, Alho CJR, Nunes LB, Haddad JV. 2010 Injuries and envenoming by aquatic animals in fishermen of Coxim and Corumbá municipalities, State of Mato Grosso do Sul, Brazil: identification of the causative agents, clinical aspects and first aid measures. Medicina Tropical (Minas Gerais) [periódico na Internet]. 2010 [citado em 02 de jan. 2018]; 43(5): 486-490. Disponível em:http://www.repositorio.unb.br/bitstr eam/10482/10603/1/ARTIGO_Injuries EnvenomingAquatic.pdf

22. Negreiros MMB, Yamashita $\mathrm{S}$, Sardenberg T, Fávero JEL, Ribeiro FAH, Haddad JWT, Haddad JV. Diagnostic imaging of injuries caused by venomous and traumatogenic catfish. Medicina Tropical (Minas Gerais) [periódico na Internet]. 2016 [citado em jan. 15]; 49(4): 530-533. Disponível em: http:/www.scielo.br/ pdf/rsbmt/v49n4/1678-9849-rsbmt-4904-00530.pdf. 
23. Garrone ND, Haddad JV. Arraias em rios da região Sudeste do Brasil: locais de ocorrência e impactos sobre a população. Medicina Tropical (Minas Gerais) [periódico na Internet]. 2010 [citado em abr. 25]; 43(1), 82-88. Disponível em: http:/www.scielo.br/ $\mathrm{pdf} / \mathrm{rsbmt} / \mathrm{v} 43 \mathrm{n} 1 / \mathrm{a} 18 \mathrm{v} 43 \mathrm{n} 1 . \mathrm{pdf}$.

24. Rosa MFM, Mattos UAO. A saúde e os riscos dos pescadores e catadores de caranguejo da Baía de Guanabara. Ciência e Saúde Coletiva (Rio de Janeiro) [periódico na Internet]. 2010 [citado em jan. 18]; 15(1): 1543-1552. Disponível em: http://www.scielo.br/ pdf/csc/v15s1/066.pdf.

25. Doimo RAF, Barella W, Mello ALR, Ramires MA. importância do uso de equipamentos de proteção individual para a redução de acidentes no trabalho dos pescadores artesanais da Baixada Santista. Ciência Social (São Paulo) [periódico na Internet]. 2013 [citado 2018 abr. 13]; 2(1): 4853. Disponível em: periodicos.unisa nta.br/index.php/lss/article/download/1 84/16353-71. 